\title{
Performance of a recursive MBER decision feedback equalizer in long multipath channel
}

\author{
G. C. Chunga) \\ Faculty of Engineering, Multimedia University Jalan Multimedia, \\ 63100 Cyberjaya, Selangor, Malaysia \\ a) gcchung@mmu.edu.my
}

\begin{abstract}
Minimum bit error rate (MBER) criterion is formulated to minimize the bit-error-rate (BER) of a communication system, which is the true performance indicator in channel equalization. However, MBER decision feedback equalizer (DFE) undergoes slow convergence speed, and BER misadjustment in long multipath channel as demonstrated in this paper. We propose a MBER-DFE receiver adopted with recursive least square (RLS) adaptive algorithm. Results showed that the proposed receiver can achieve faster convergence rate and considerable performance as compared to conventional MBER receiver in a realistic long ultra-wideband (UWB) multipath channel.
\end{abstract}

Keywords: minimum bit error rate, bit error rate, decision feedback equalizer, recursive least square, ultra wideband

Classification: Microwave and millimeter wave devices, circuits, and systems

\section{References}

[1] S. R. D. Paulo, Adaptive Filtering Algorithms and Practical Implementation, Springer, USA, 2008.

[2] S. Chen, "Adaptive Minimum Bit-Error-Rate Filtering," IEEE Proceedings - Vision, Image and Signal Processing, vol. 151, no. 1, pp. 76-85, Feb. 2004.

[3] C. C. Yeh, R. R. Lopes, and J. R. Barry, "Approximate Minimum BitError Rate Multiuser Detection," Proc. Globecom, Sydney, pp. 3590-3595, Nov. 1998

[4] S. Chen, A. L. Samingan, and B. Mulgrew, "Adaptive Minimum-BER Linear Multiuser Detection for DS-CDMA Signals in Multipath Channels," IEEE Trans. Signal Process., vol. 49, no. 6, pp. 1240-1247, June 2001.

[5] B. Mulgrew and S. Chen, "Adaptive Minimum-BER Decision Feedback Equalisers for Binary Signalling," IEEE Trans. Signal Process., vol. 81, no. 7, pp. 1479-1489, 2001.

[6] J. R. Foerster, "Channel Modeling Sub-committee Report Final," IEEE 802.15.SG3a, Dec. 2002.

[7] J. R. Foerster, "Effect of Multipath Inteference on the Performance of UWB Systems in an Indoor Wireless Channel," Proc. Vehicular Technology Conference, vol. 2, pp. 1176-1180, May 2001. 
[8] C. R. Anderson, J. H. Reed, R. M. Buehrer, D. Sweeney, and S. Griggs, Introduction to Ultra Wideband Communication Systems, Prentice Hall, USA, 2005.

\section{Introduction}

In digital communication, adaptive equalization is a common technique used for channel estimation and interference mitigation, such as inter-symbol interference (ISI) and multiple-access interference (MAI) [1]. Such equalization technique requires optimization criterion such as maximum likelihood sequence estimation (MLSE), and minimum mean square error (MMSE). Due to the high complexity in MLSE receiver, MMSE is the most popular methods used for channel equalization. However, studies show that the biterror-rate (BER) is the true performance indicator and therefore minimum bit error rate (MBER) criterion is formulated [2].

MBER receiver has been shown to outperform MMSE receiver significantly. A least mean square (LMS)-style stochastic gradient adaptive MBER criterion is proposed and the algorithm has the similar low-complexity computation as the LMS algorithm [2]. An alternative of MBER criterion, called the stochastic approximate minimum bit error rate (AMBER) has been shown to achieve comparable BER performance to the stochastic gradient MBER criterion with less computation complexity than the LMS algorithm [3]. However, AMBER algorithm requires large amount of training data to obtain optimal BER. In [4], a new LMS-style stochastic MBER, which is the least bit error rate (LBER), has been developed. Simulation results indicate that the LBER receiver achieves significant performance gain compared to the MMSE receiver in multipath channel. On the other hand, MBER criterion has been studied in DFE [5], and found to have slow convergence speed too. Under this circumstance, MBER algorithm may not converge to a point where it can separate the noises correctly.

Therefore, we attempt to study the performance of the LMS-style MBERDFE receiver in severe multipath channel in terms of convergence speed and BER misadjustment in this paper. We choose UWB channel in our analysis since a typical high data rate UWB indoor channel response usually contains 60-200 multipaths [6], which cause severe intersymbol interferences (ISI) even in a single user environment [7]. In long multipath channel, slow convergence rate and BER misadjustment may occur due to the presence of feedback propagation errors in DFE. We develop a low complexity recursive MBER-DFE receiver to overcome this problem. Finally, we analyze the BER performance, and study the convergence rate and the BER misadjustment of the proposed receiver. 


\section{System model}

In this paper, we have chosen UWB as a transmission channel in our system design since it has long multipath channel characteristic. A simple binary phase shift keying (BPSK) UWB system transmits extremely short pulses in the form of [8],

$$
x(t)=\sum_{i=-\infty}^{\infty} b^{\prime}[i] p\left(t-i T_{s}\right)
$$

where $\left\{b^{\prime}[i]\right\} \in\{-1,1\}$ represents the BPSK bit stream and $p(t)$ is the UWB pulse with a symbol duration, $T_{s}$. The UWB has a standard multipath channel model defined in the IEEE P802.15.3a Wireless Personal Area Networks [6]. This UWB channel model is derived from the Saleh-Valenzuela model with slight modifications. The received signal after passing through the multipath channel is given as [8],

$$
r(t)=\sum_{i=-\infty}^{\infty} b^{\prime}[i] \sum_{m=0}^{M-1} h^{\prime}[m] p\left(t-i T_{s}-\tau_{m}\right)+n(t)
$$

where $h^{\prime}[m]$ is the UWB impulse response, $n(t)$ is the additive white Gaussian noise (AWGN) with zero mean and variance $N_{0} / 2 . M$ is the number of paths in the multipath channel and $\tau$ is the multipath delays. The received signal is then filtered by a DFE [1] and the output of a $k^{\text {th }}$ detected symbol is:

$$
y^{\prime}[k]=g^{T} r[k]-h^{T} z[k-D]
$$

where $g^{T}=\left[g_{0}, g_{1} \cdots g_{u-1}\right]$ and $h^{T}=\left[h_{0}, h_{1} \cdots h_{v-1}\right]$ are the feedforward coefficients and feedback coefficients respectively. The $u$ and $v$ are the length of the feedforward and feedback equalizer respectively. $r[k]=\left[r_{k}, r_{k+1} \cdots\right.$ $\left.r_{k+u-1}\right]$ are the received symbols and $z[k-D]=\left[z_{k-D}, z_{k+1-D} \cdots z_{k+v-1-D}\right]$ are the previously detected symbols with delay $D$. The final detected $k^{t h}$ symbol is obtained by making a hard decision on $y^{\prime}[k]$ :

$$
z^{\prime}[k]=\operatorname{sgn}\left(y^{\prime}[k]\right) .
$$

\section{MBER decision feedback equalization}

In order to reduce the computation of simulation, a simplified stocastic MBER criterion, named as AMBER [3] is used. Using the LMS adaptive algorithm, the adaptive AMBER-DFE can be illustrated as,

$$
\begin{aligned}
& g_{k+1}=g_{k}+\mu_{f} I_{k} z^{\prime}[k] r[k] \\
& h_{k+1}=h_{k}+\mu_{b} I_{k} z^{\prime}[k] r[k]
\end{aligned}
$$

where $\mu_{f}$ is the feedforward LMS step size and $\mu_{b}$ is the feedback LMS step size. $I_{k}$ is the indicator function with non-negative threshold $\tau$ given as,

$$
I_{k+1}=\frac{1}{2}\left(1-\operatorname{sgn}\left(z^{\prime}[k] y^{\prime}[k]-\tau\right)\right) .
$$

The purpose of having the threshold parameter $\tau$ is to increase the convergence speed for adaptation of the receiver. 


\subsection{Recursive MBER decision feedback equalizer}

In this paper, we propose an alternative of MBER criterion using the simplified deterministic recursive RLS adaptive algorithm [1], called recursive MBER (RMBER). The modified RMBER-DFE can be described as,

$$
\begin{aligned}
& g_{k+1}=g_{k}+P_{f, k} I_{k} z^{\prime}[k] \\
& h_{k+1}=h_{k}+P_{b, k} I_{k} z^{\prime}[k]
\end{aligned}
$$

where $P_{f}$ and $P_{b}$ are the inverse of the deterministic correlation matrix for the feedforward and feedback input signals and are given as,

$$
\begin{aligned}
P_{f, k+1} & =\frac{1}{\lambda}\left(P_{f, k}-\frac{P_{f, k} \psi_{f} r[k]}{\lambda+\psi_{f} r[k]}\right) \\
P_{b, k+1} & =\frac{1}{\lambda}\left(P_{b, k}-\frac{P_{b, k} \psi_{b} r[k]}{\lambda+\psi_{b} r[k]}\right)
\end{aligned}
$$

where $\lambda$ is an exponential factor that should be chosen in the range $0 \leq$ $\lambda \leq 1$, and $\psi_{f}=P_{f, k} r[k]^{T}$ and $\psi_{b}=P_{b, k} r[k]^{T}$ are the auxiliary vectors for the feedforward and feedback signals required to reduce the computation complexity. The initial value of $P_{f}$ and $P_{b}$ are expressed as,

$$
\begin{aligned}
& P_{f}=\frac{1}{\eta_{f}} \Phi \\
& P_{b}=\frac{1}{\eta_{b}} \Phi
\end{aligned}
$$

where $\eta_{f}$ is the feedforward RLS step size and $\eta_{b}$ is the feedback RLS step size. $\Phi$ is the matrix identity.

\section{Results and discussion}

In this section, we present simulation results for five different receivers: the linear MMSE, non-linear MMSE-DFE, AMBER-DFE, LBER-DFE and the proposed RMBER-DFE. We evaluate the BER performance and the convergence rate of the above receivers using UWB multipath channel model 1 (CM1) and channel model 4 (CM4) [6].

The feedforward LMS step sizes $\mu_{f}$ used for the MMSE and MMSE-DFE are 0.01 for fair comparisons. The feedback LMS step sizes $\mu_{b}$ of the MMSEDFE, AMBER-DFE and LBER-DFE receivers and, the feedback RLS step size $\eta_{b}$ of the RMBER-DFE receiver are chosen as 0.0001 and 10000 respectively to minimize propagation feedback errors. Besides that, the thresholds $\tau$ used for the AMBER-DFE and RMBER-DFE are 0.5 and the exponential factor $\lambda$ used for the RMBER-DFE is 0.98 . The tap lengths for the feedforward filter, $u$ and the feedback filter, $v$ are 7 and 1 respectively. The feedforward LMS step sizes $\mu_{f}$ of the AMBER-DFE and LBER-DFE receivers and, the feedforward RLS step sizes $\eta_{f}$ of the RMBER-DFE receiver are varied thorough the simulations for performance comparisons. 


\subsection{BER performance comparisons}

Fig. 1 and 2 shows the BER performance of five different receivers at a data rate of $150 \mathrm{Mbps}$ under CM1 and $35 \mathrm{Mbps}$ under CM4 respectively. In Fig. 1, the AMBER-DFE, LBER-DFE and the proposed RMBER-DFE receivers outperform the performances of the MMSE and MMSE-DFE receivers under CM1. Notice that the MMSE-DFE achieves better BER performance than the linear MMSE receiver. At a BER of $10^{-4}$, both the LBER-DFE receivers with $\mu_{f}=0.01$ and $\mu_{f}=0.001$ and the proposed RMBER-DFE receivers with $\eta_{f}=100$ and $\eta_{f}=1000$ have approximately the same BER performances. Both receivers achieve a gain of about $0.5 \mathrm{~dB}$ compared to the MMSE-DFE with $\mu_{f}=0.01$. However, it is well observed that the AMBERDFE receiver with $\mu_{f}=0.001$ has a substantially performance decrement of about $4 \mathrm{~dB}$ compared to the proposed receiver.

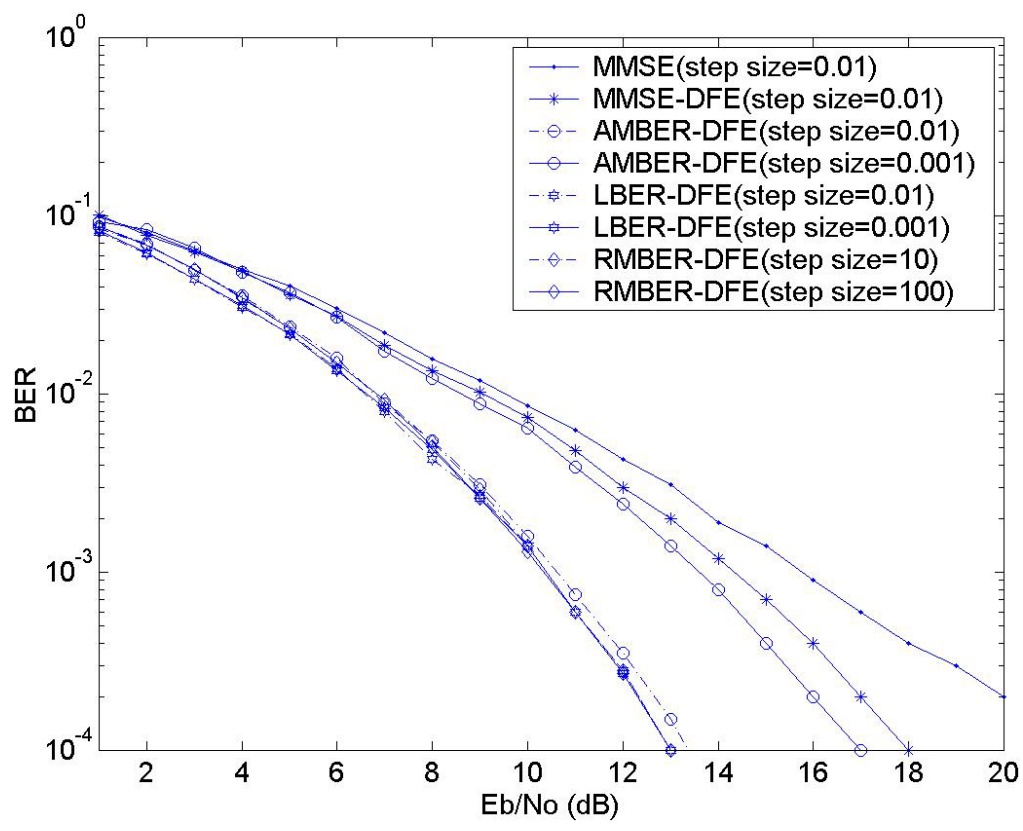

Fig. 1. BER comparison of the MMSE, MMSE-DFE, AMBER-DFE, LBER-DFE and proposed RMBER-DFE receivers in UWB channel CM1 for $150 \mathrm{Mbps}$ of data rates.

In Fig. 2, the AMBER-DFE, LBER-DFE and the proposed RMBER-DFE receivers again outperform the performances of the MMSE and MMSE-DFE receivers under CM4. At a BER of $10^{-4}$, the MMSE-DFE achieves a gain of about $0.5 \mathrm{~dB}$ compared to the linear MMSE receiver. Nonetheless, the LBER-DFE and the proposed RMBER-DFE receivers have the smiliar best BER performances. Notice that the RMBER-DFE receiver suffers slightly performance degradation when the feedforward step size increases from 100 to 1000. On the other hand, both LBER-DFE and RMBER-DFE receivers achieve a gain of about $1 \mathrm{~dB}$ and $2 \mathrm{~dB}$ compared to the MMSE-DFE with $\mu_{f}=0.01$ and $\mu_{f}=0.001$. Hence, both results show that the performance of the LBER-DFE and the proposed RMBER-DFE receivers with different feed- 


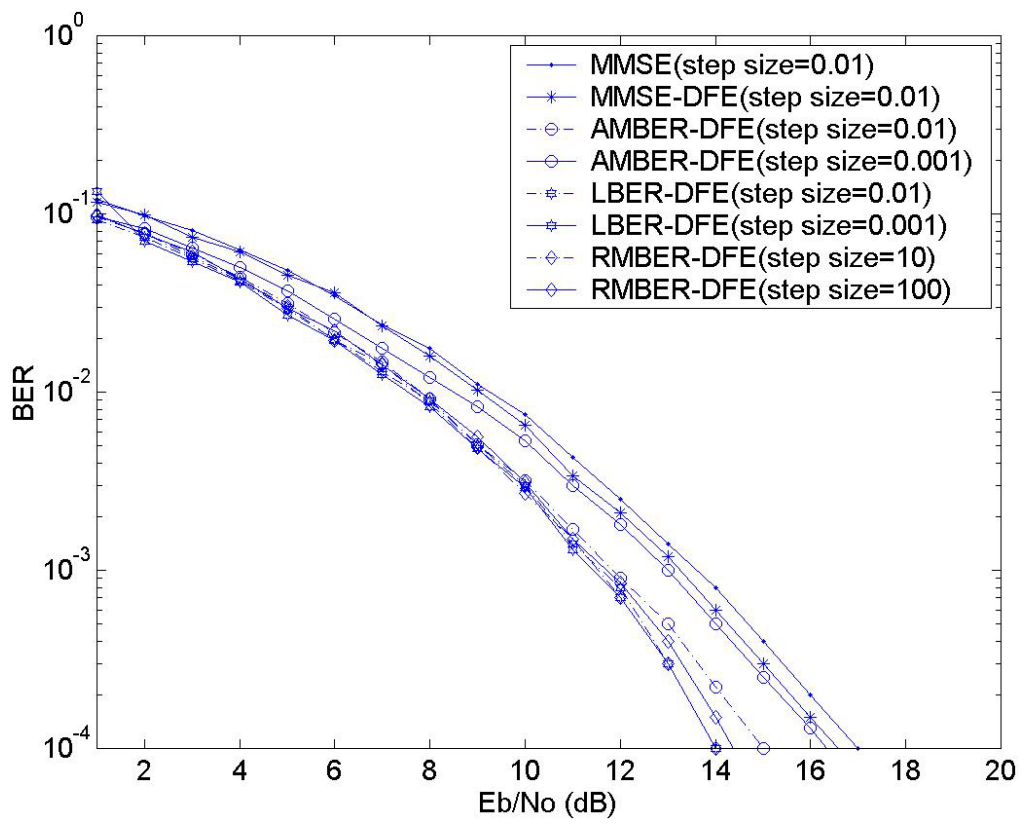

Fig. 2. BER comparison of the MMSE, MMSE-DFE, AMBER-DFE, LBER-DFE and proposed RMBER-DFE receivers under UWB channel CM4 for 35 Mbps of data rates.

forward step sizes remain superior over the AMBER-DFE receivers without suffering any BER misadjusment.

\subsection{Convergence rate}

In this section, we study the convergence rate of the AMBER-DFE, LBERDFE and the proposed RMBER-DFE at $E_{b} / N_{0}=15 \mathrm{~dB}$ under UWB channel CM1 and CM4 as presented in Fig. 3. The simulated data transfer rates are $150 \mathrm{Mbps}$ and $35 \mathrm{Mbps}$ respectively. We compare the convergence behavior by varying the feedforward LMS step sizes $\mu_{f}$ of the AMBER-DFE and LBERDFE receivers and, the feedforward RLS step sizes $\eta_{f}$ of the RMBER-DFE receivers.

In Fig. 3 (a) and (b), both the proposed RMBER-DFE with $\eta_{f}=100$ and $\eta_{f}=1000$ have the fastest convergence rates. They reach the optimum BER in less than 500 of iteration. On the other hand, the AMBER-DFE receivers with $\mu_{f}=0.01$ and $\mu_{f}=0.001$ require approximately 750 of iteration and 2500 of iteration respectively under CM1 and approximately 500 of iteration and 2500 of iteration respectively under CM4. However, the LBER-DFE receivers suffer the slowest convergence rate in long multipath channel. The LBER-DFE receivers with $\mu_{f}=0.01$ require approximately 2000 of iteration under CM1 and 1000 of iteration under CM4 respectively. Notice that the LBER-DFE receivers with $\mu_{f}=0.001$ require more than 2500 of iteration under both channels. Hence, both results show that the proposed receivers remain fast convergence rate with different feedforward step sizes compared to the AMBER-DFE and LBER-DFE receivers. 


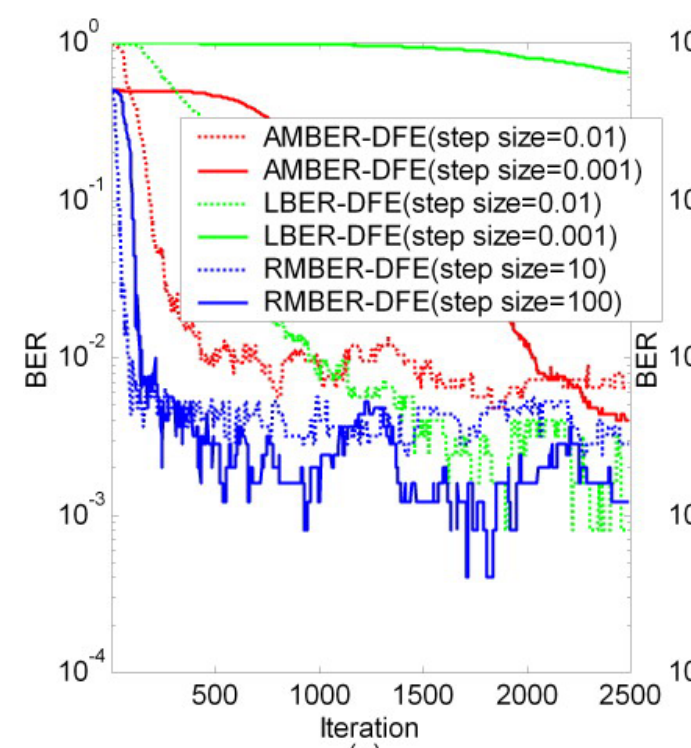

(a)

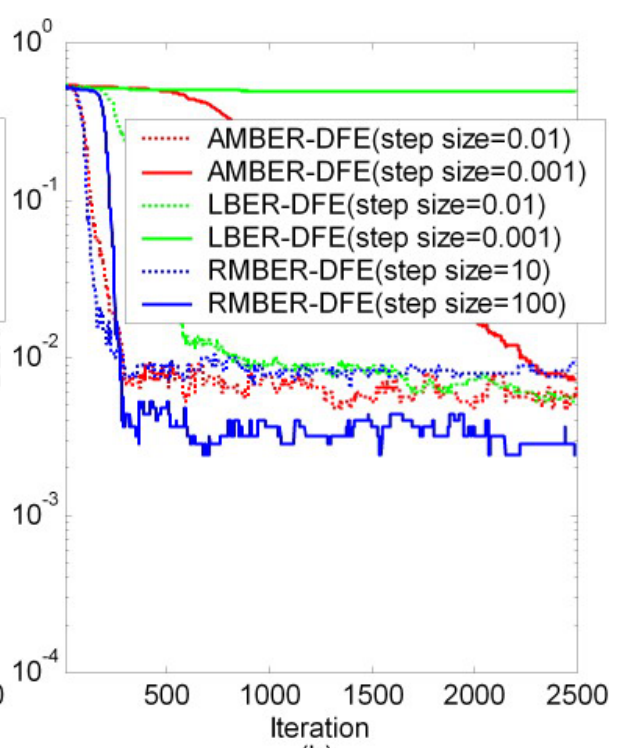

(b)

Fig. 3. Convergence comparison of the AMBER-DFE, LBER-DFE and proposed RMBER-DFE under UWB channel (a) CM1 and (b) CM4.

\section{Conclusion}

A recursive least square receiver using the MBER criterion has been proposed in a typical long multipath channel to mitigate ISI. It has been presented that the proposed RMBER-DFE receiver is capable of achieving significant BER performance gains against the AMBER-DFE receiver while obtaining faster convergence rate compared to the LBER-DFE receiver with different variation of feedforward step size. Therefore, the proposed receiver has fast convergence rate as well as considerable BER performance. 